\title{
Geometrically-controlled twist transitions in nematic cells
}

\author{
Pedro Patrício ${ }^{1}$, M.M. Telo da Gama ${ }^{1}$, and S. Dietrich ${ }^{2,3}$ \\ 1 Departamento de Física da Faculdade de Ciências and Centro de Fúsica Teórica e Computacional \\ Universidade de Lisboa, Avenida Professor Gama Pinto 2, P-1649-003 Lisboa Codex, Portugal \\ ${ }^{2}$ Max-Planck-Institut für Metallforschung, Heisenbergstr. 1, D-70569 Stuttgart, Germany \\ ${ }^{3}$ Institut für Theoretische und Angewandte Physik, Universität Stuttgart, Pfaffenwaldring 57, D-70569 Stuttgart, Germany
}

(February 2002)

\begin{abstract}
We study geometrically-controlled twist transitions of a nematic confined between a sinusoidal grating and a flat substrate. In these cells the transition to the twisted state is driven by surface effects. We have identified the mechanisms responsible for the transition analytically and used exact numerical calculations to study the range of surface parameters where the twist instability occurs. Close to these values the cell operates under minimal external fields or temperature variations.
\end{abstract}

PACS numbers: $61.30 . \mathrm{Cz}, 61.30 . \mathrm{Gd}$

Liquid-crystal (LC) displays consist of a LC layer confined between two surfaces that impose a preferred orientation of the average molecular direction $\mathbf{n}(\mathbf{r})$. Current nematic displays rely on voltage-induced reorientation of the director within the bulk LC layer. Recently, textured surfaces (on scales of the order of $1 \mu \mathrm{m}$ ) were designed for patterned alignment of LC [1 1 , opening possibilities for an improved performance of LC cells. In view of the very rich behavior of LCs even on homogeneous substrates [5] and of simple fluids on structured surfaces [6] such technological advances beyond a trial and error procedure require theoretical guidance. As a first step in this direction we establish the minimal model appropriate to the theoretical description of patterned LC cells and develop an efficient method of solving it. This study contributes to the broader perspective of how well-defined structures on solid surfaces can be imprinted on adjacent soft matter. The particular softness of LCs leads to the expectation of very pronounced effects.

The microscopic description of LC surfaces and interfaces is complex. The degree of nematic order, biaxility, etc. vary in the interfacial region over molecular distances while the director may vary over macroscopic distances. In LC displays, the characteristic distance over which the director varies is set by the cell dimensions or by the electric correlation length $[7]$. Under most experimental conditions the length over which the director varies is of the order of $\mu \mathrm{m}$ and a macroscopic or elastic theoretical description is adequate [7,8]. The macroscopic (second order) elastic free energy of bulk nematics was established more than 40 years ago [9] but the status of the surface contributions in the weak anchoring regime is still controversial 10.11].

In the following we propose and minimize an elastic free energy for patterned LC displays that includes bulk and surface terms. We consider the twist cell proposed in a recent experiment, where the nematic is confined between a flat and a sinusoidal grating surface [1]. In the experiment a voltage-controlled twist (VCT) effect highly sensitive to the surface properties of the cell (grating geometry and anchoring strength) has been reported, for gratings on the scale of tenths of $\mu \mathrm{m}$. Berreman 12] and de Gennes [7] first considered grating surfaces to explain azimuthal anchoring by elastic effects only. Faetti [13], and more recently Fournier and Galatola [14], generalized the effective azimuthal anchoring energy by introducing local anchoring at the grating surface. Barbero and Durand [15] also considered grating surfaces with characteristic lengths comparable to the nematic correlation length. They used the Landau-de Gennes free energy [7] to describe the induced quasi-melting caused by the rough surface. Over the last few years there has been a considerable surge of interest in the influence of gratinglike surfaces on the structural properties of LCs because new techniques enabled the manufacture of controlled undulated surfaces, allowing for meaningful comparisons between theory and experiments. Among these new systems the aforementioned twist displays exhibit excellent viewing angle characteristics [1], that are important for technological applications.

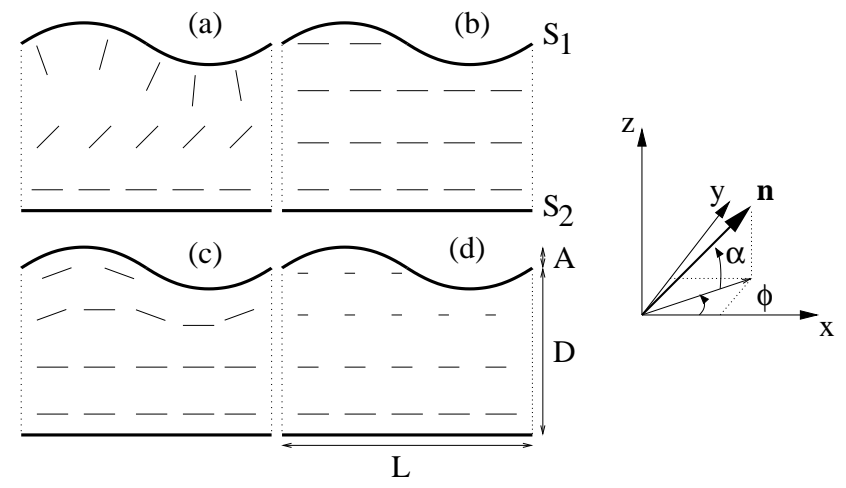

FIG. 1. Nematic director field (projected onto the xz plane) for increasing anchoring strengths $W_{1}$. (a) Distorted director field, for strongly negative anchoring strength $W_{1}$. (b) Upon increasing $W_{1}$ a first instability leads to a nearly uniform director field. (c) For larger $W_{1}$ the director field follows the orientation of the surface. (d) A second instability induces a twisted director field. 
In spite of its importance, a theoretical study of twist cells covering the whole range of operating parameters is still lacking. In order to explore how variations in the geometry of the cell can induce the reorientation of the bulk nematic director we consider the total free energy of the LC cell as the sum of a bulk elastic free energy $F_{b}$ and a surface contribution $F_{s}$. The former is the Frank elastic free energy [7]

$$
\begin{aligned}
F_{b} & =\frac{1}{2} \int_{V}\left\{K_{11}(\nabla \cdot \mathbf{n})^{2}+K_{22}[\mathbf{n} \cdot(\nabla \times \mathbf{n})]^{2}\right. \\
& \left.+K_{33}[\mathbf{n} \times(\nabla \times \mathbf{n})]^{2}\right\} d^{3} \mathbf{r}
\end{aligned}
$$

where $K_{11}, K_{22}$, and $K_{33}$ are the elastic constants associated with splay, twist, and bend distortions, respectively. $F_{s}$ includes the anchoring energy for which we adopt the Rapini-Papoular form 16]

$$
F_{s}=\frac{W_{1}}{2} \int_{S_{1}}(\mathbf{n} \cdot \nu)^{2} d^{2} \mathbf{r}+\frac{W_{2}}{2} \int_{S_{2}}(\mathbf{n} \cdot \nu)^{2} d^{2} \mathbf{r} .
$$

These integrals run over the two cell surfaces and $W_{i}$, $i=1,2$, is the corresponding anchoring strength that characterizes each surface. $\nu$ is the local unit vector normal to the surface. For negative $W_{i}$ this energy contribution favors normal surface orientation of the nematic while positive $W_{i}$ favor planar (degenerate) orientation at the surface. For inhomogeneous substrates in the weak anchoring regime one may have to include a surface elastic term associated with the saddle-splay distortion [10]. Its contribution will be considered later.

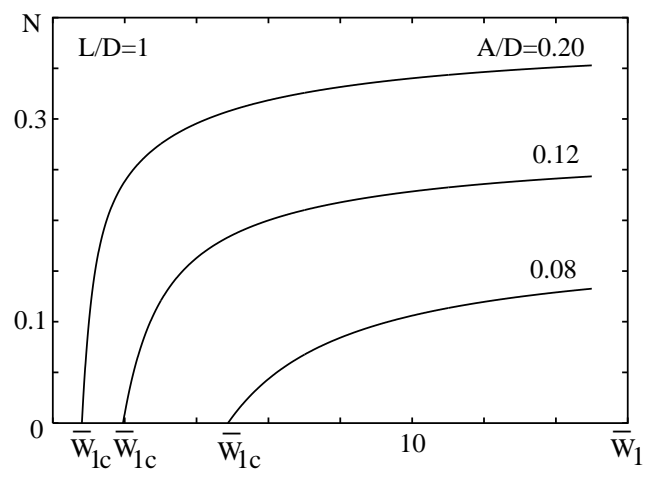

FIG. 2. The twist instability (numerical results). The twist order parameter $N=D^{-3} \int_{V} \mathbf{n}_{y}^{2} d^{3} \mathbf{r}$ as a function of the reduced anchoring strength $\bar{W}_{1}=W_{1} D / K_{11}$ for different groove depth $A / D$. To favor twist configurations we used $K_{11}=K_{33}=2 K_{22} . N$ vanishes linearly at $\bar{W}_{1 c}$.

The total free energy, $F_{t}=F_{b}+F_{s}$, is a functional of the two angles $\alpha(\mathbf{r})$ and $\phi(\mathbf{r})$, characterizing the nematic director $\mathbf{n}=(\cos \alpha \cos \phi, \cos \alpha \sin \phi, \sin \alpha)$. The cells considered in this letter consist of a nematic LC confined between a sinusoidal grating $(z=D+A \sin q x$, where $q=2 \pi / L$ and $A$ is the groove depth) and a flat substrate $(z=0)$ (see Fig. 1). In Ref. [1] the surfaces were treated so that the grating induces (weak) homeotropic, i.e., normal anchoring with respect to the local surface direction, while the flat surface induces (strong) homogeneous uniaxial anchoring in the direction perpendicular to the grooves. In the following calculations we keep the homogeneous strong anchoring condition at the flat surface $(\alpha(x, z=0)=\phi(x, z=0)=0)$ but we vary the anchoring strength $W_{1}$ (including its sign) at the grating surface. Finally, we use periodic boundary conditions $\alpha(x=0, z)=\alpha(x=L, z)$ and $\phi(x=0, z)=\phi(x=L, z)$.

Figure 1 illustrates the behavior of a confined LC with a small twist elastic constant as the anchoring strength $W_{1}$ increases. For large negative values of $W_{1}$, the grating surface induces normal orientation of the nematic. Upon increasing $W_{1}$ a first instability occurs so that the director field becomes nearly uniform resulting from the competition of two effects: the anchoring energy favoring homeotropic anchoring, and the elastic energy favoring homogeneous alignment at the grated surface. The critical anchoring strength for a rectangular cell $D \times S$, where $\alpha(x, z)=\alpha z / D$, is found easily. For small $\alpha$, the total free energy is $F_{t}=(S / 2)\left(W_{1}+K_{11} / D\right) \alpha^{2}$, where $S$ is the area of the flat cell surface. The director field is uniform when the coefficient of $\alpha^{2}$ is positive, i.e., for $W_{1}>-K_{11} / D$. In the limit of small groove depth $A$ the corrections arising from the grating are found by first-order perturbation analysis about the rectangular cell. When $W_{1}$ becomes positive, the nematic orientation changes continuously to follow the sinusoidal shape of the boundary. Beyond a certain threshold, the bulk bending energy of this deformation is comparable with a bulk twist deformation and a new instability occurs. Owing to the degeneracy of the planar anchoring, the sinusoidal and the twisted configuration have approximately the same surface free energy.

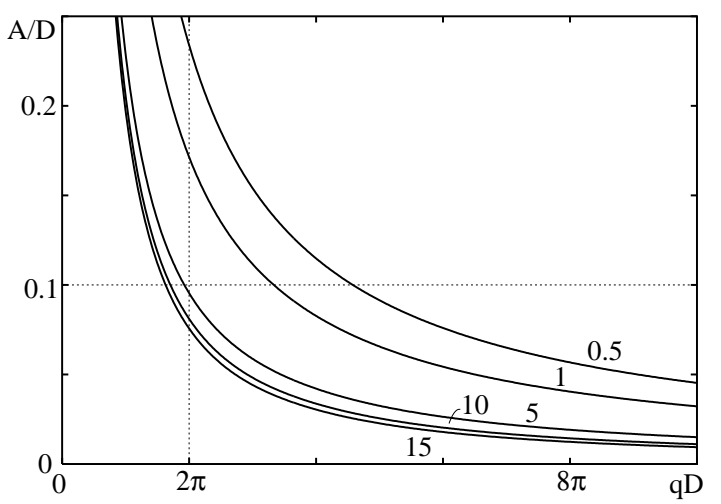

FIG. 3. The onset of the twist instability occurs for anchoring strengths $W_{1 c}$ which depend on the cell geometry $(A, q=2 \pi / L)$. Here the contour lines for $\bar{W}_{1 c}=0.5,1,5,10$, and 15 are shown for $K_{11}=K_{33}=2 K_{22}$. These results are obtained analytically based on Eq.(9). The vertical and horizontal line indicates systems with $L=D$ or $A=D / 10$, respectively (c.f., Fig. 4 (b),(c)). 
The twist instability depends strongly on the geometry of the cell. In Fig. 2 we plot the numerical results for the twist order parameter $N=D^{-3} \int_{V} \mathbf{n}_{y}^{2} d^{3} \mathbf{r}$ as a function of the anchoring strength $W_{1}$ for cells with different groove depth $A$.

The free energy of the sinusoidal configuration $F_{\text {sin }}$ is estimated by using the variational ansatz

$$
\alpha_{\sin }(x, z)=\mathcal{B} A q \cos q x \frac{\sinh \lambda z}{\sinh \lambda D}
$$

This function respects the boundary conditions and has two parameters $\mathcal{B}$ and $\lambda$. If the nematic director field is nearly uniform the dimensionless parameter $\mathcal{B} \approx 0$. On the contrary, if the director is parallel to the sinusoidal surface, $\mathcal{B}=1 . \lambda^{-1}$ defines the distance over which the nematic deformation is influenced by the grating surface. For small groove depth $A / D$ the total energy is

$$
F_{\text {sin }} \approx \frac{S}{4}(A q)^{2}\left[Y(\lambda) \mathcal{B}^{2}+W_{1}(1-\mathcal{B})^{2}\right]
$$

where $Y(\lambda)=K_{11} \lambda^{2} F_{1}(\lambda)+K_{33} q^{2} F_{2}(\lambda)$, with $F_{1}=\int_{0}^{D}(\cosh \lambda z / \sinh \lambda D)^{2} d z$ and $F_{2}=$ $\int_{0}^{D}(\sinh \lambda z / \sinh \lambda D)^{2} d z$. Minimization with respect to the variational parameters yields $\lambda=q \sqrt{K_{33} / K_{11}}$ and $\mathcal{B}=W_{1} /\left(W_{1}+Y\right)$. The total free energy follows as

$$
F_{\text {sin }} \approx \frac{S}{4}(A q)^{2} \frac{Y W_{1}}{Y+W_{1}} .
$$

For $W_{1} / Y \gg 1$ and $\lambda D \gg 1$ we recover the Berreman energy

$$
F_{\text {sin }} \approx \frac{S}{4}(A q)^{2} q \sqrt{K_{33} K_{11}}
$$

which is independent of the cell size $D$. However, when the flat surface approaches the grating one finite size effects come into play. In this case, for $\lambda D \ll 1$, $Y \approx\left[K_{11}+K_{33}(q D)^{2} / 3\right] / D$.

In order to estimate the critical anchoring strength $W_{1 c}$, i.e., the threshold for the twist instability, we take $\phi(x, z)=\phi z / D$ and use an expansion for the total energy in powers of $\phi$ :

$$
F_{t}[\alpha, \phi]=F_{t}^{(0)}[\alpha]+\phi^{2} F_{t}^{(2)}[\alpha]+\ldots .
$$

The sinusoidal deformation $\alpha_{\sin }$ (with $\phi=0$ ) minimizes the free energy if the coefficient $F_{t}^{(2)}\left[\alpha_{\text {sin }}\right]$ is positive. Supposing that $K_{22} / K_{11} \sim(A / D)^{2}$ is small one has

$$
F_{t}^{(2)} \approx \frac{S K_{22}}{2 D}-\frac{S}{4}(A q)^{2} \frac{Y W_{1}}{Y+W_{1}}
$$

The twist instability occurs for the threshold value given by $F_{t}^{(2)}\left[\alpha_{\text {sin }}\right]=0$, or equivalently when the energy of the sinusoidal deformation equals

$$
F_{s i n}^{(c)}=\frac{S K_{22}}{2 D}
$$

In Fig. 3 we plot the results obtained from solving this equation for different cell geometries $(A, q=2 \pi / L)$. To favor twist configurations we used $K_{11}=K_{33}=2 K_{22}$.

In order to overcome the limitations of the above analytic analysis extensive numerical calculations of cells with a wide range of surface parameters (groove depth, pitch, and anchoring strength) have been carried out. The calculation of the twist transition requires a numerical procedure capable of evaluating the bulk and surface contributions to the free energy very accurately. Owing to the geometrical pattern of the grating surface this turns out to be a challenging numerical problem. We used finite-element discretizations of the functions $\alpha(x, z)$ and $\phi(x, z)$ (dividing the space into small triangles where the functions are approximated linearly) and found the minimum of the free energy by standard numerical techniques. Non-uniform adaptive meshes have been used: finer meshes were required close to the grating surface where the fields vary more rapidly [17]. In addition, a finite-element triangulation that approximates as closely as possible the geometrical boundary of the cell was required since the critical anchoring strength depends sensitively on these surface terms.

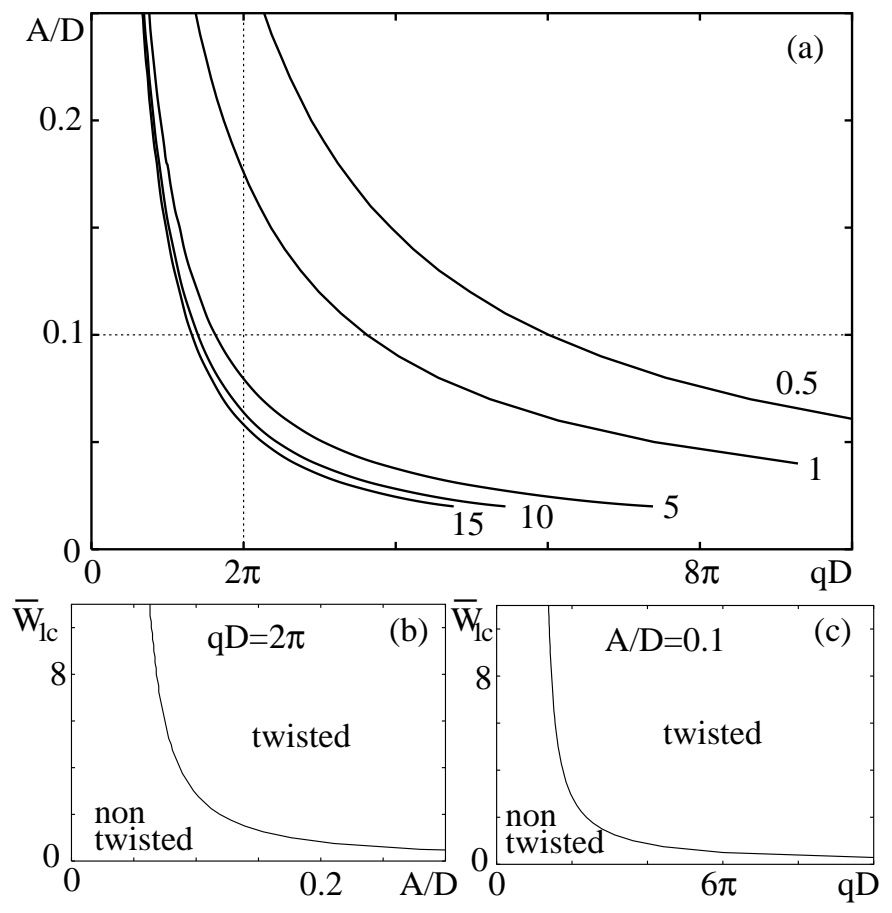

FIG. 4. Critical anchoring strength $W_{1 c}(A, q)$ for $K_{11}=K_{33}=2 K_{22}$ as in Fig. 3 obtained from an exact numerical solution. (a) Contour lines $\bar{W}_{1 c}=0.5,1,5,10$, and 15. (b) $\bar{W}_{1 c}(A, L=D)$. (c) $\bar{W}_{1 c}(A=0.1 D, q)$. 
These numerical results are shown in Fig. 4. From this figure one infers that $W_{1 c}$ diverges for a set of the geometrical parameters of the cell. This set defines a range of parameters for which the nematic orientation will never twist. Outside this region, for larger values of $A$ and $q$, a twisted nematic director may occur for small values of $\bar{W}_{1 c}=W_{1 c} D / K_{11}$.

For completeness, we consider the elastic term associated with the saddle-splay distortion neglected in the previous analysis. This term is usually written in the form

$$
F_{s s}=K_{24} \int_{V} \nabla \cdot[(\mathbf{n} \cdot \nabla) \mathbf{n}-\mathbf{n}(\nabla \cdot \mathbf{n})] d^{3} \mathbf{r}
$$

which reveals its surface nature. The value of the surface elastic constant $K_{24}$ is bounded for stability reasons. In fact, the bulk free energy in Eq.(1)) is the sum of quadratic terms and it is well defined only if the bulk elastic constants $K_{11}, K_{22}$, and $K_{33}$ are positive. A stability analysis of the surface energy in Eq.(10) yields the constraint $0<K_{24}<\min \left(K_{11}, K_{22}\right)[18]$. In Fig. 5 we plot the numerical solutions for $\alpha(x, z)$ for physical and unphysical values of $K_{24}$. By varying $K_{24}$ (between 0 and $K_{22}$ ) we found that the thresholds $W_{1 c}$ for the twist instability are not significantly affected by this term [19] within the physically relevant range.
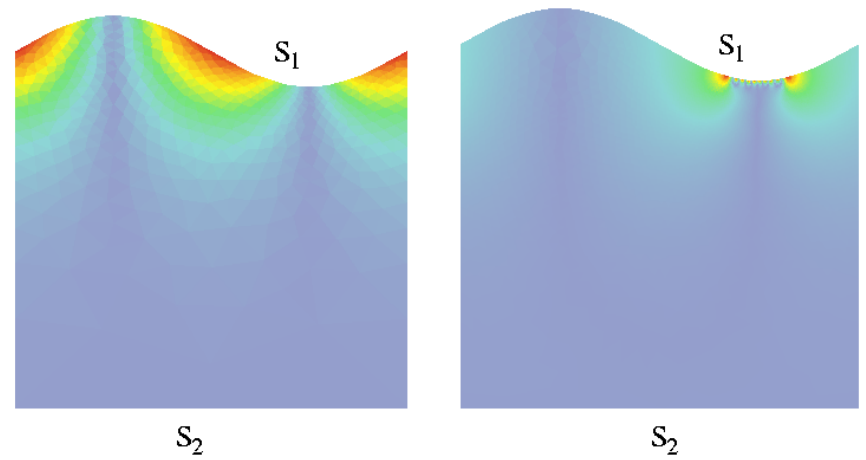

FIG. 5. Numerical solutions for the system considered above (with $A / D=0.1, q D=2 \pi, \bar{W}_{1}=2$, and $\left.K_{11}=K_{33}=2 K_{22}\right)$. The function $|\alpha(x, z)|$ is represented by a color code in which blue and red correspond to $\alpha=0$ and $\alpha=\max |\alpha|$, respectively, for physical (left: $K_{24}=0$ ) and unphysical values of $K_{24}$ (right: $K_{24}=2 K_{11}$ ). The unphysical solution on the right exhibits strong, spurious deformations close to the boundary leading to a divergent negative free energy that is bounded numerically due to the mesh discretization. The left panel corresponds to the bent sinusoidal configuration shown in Fig. 1(c).

Beyond the obvious technological importance of the twist cell - it has been shown to possess an electro-optic response far less dependent on viewing angle than other LC display configurations [1] - the system turns out to be very interesting also from a theoretical point of view: for a given anchoring strength, the twist transition is driven in the absence of an external field by the surface morphology $(A / D, q D)$. Close to the surface transition, an arbitrarily small external field or temperature variation will be sufficient to induce the reorientation of the nematic.

An obvious extension of this work includes application of a voltage between conducting substrates. This problem, however, requires special care. At constant voltage the system is no longer isolated and the minimum principle for the total free energy (with electric and elastic terms) does not apply. The free energy is minimal with respect to the nematic director field and maximal with respect to the elecric potential [20]. A generalization of the numerical method described above may not converge and a numerical solution of the Lagrangian differential equations appears to be required.

PP acknowledges the support of the Fundação para a Ciência e Tecnologia (FCT) through Grant No. SFRH/BPD/5664/2001.

[1] G. P. Bryan-Brown, C. V. Brown, I. C. Sage, and V. C. Hui, Nature 392, 365 (1998).

[2] V. K. Gupta and N. L. Abbott, Science 276, 1533 (1997).

[3] B.-W. Lee and N. A. Clark, Science 291, 2576 (2001).

[4] R. R. Shah and N. L. Abbott, Science 293, 1296 (2001).

[5] B. Jérome, Rep. Prog. Phys., 54, 391 (1991)

[6] S. Dietrich, in New Approaches to Old and New Problems in Liquid State Theory, edited by C. Caccamo, J. P. Hansen, and G. Stell (Kluwer, Dordrecht, 1999), NATOASI, Vol. C529, p. 197.

[7] P. G. de Gennes and J. Prost, The Physics of Liquid Crystals, 2nd ed. (Clarendon, Oxford, 1993).

[8] P. M. Chaikin and T. C. Lubensky, Principles of condensed matter physics (Cambridge University, Cambridge, 1995).

[9] F. C. Frank, Discuss. Faraday Soc. 25, 19 (1958).

[10] H. Yokoyama, Phys. Rev. E 55, 2938 (1997).

[11] J.-B. Fournier and P. Galatola, Phys. Rev. Lett. 82, 4859 (1999).

[12] D. W. Berreman, Phys. Rev. Lett. 28, 1683 (1972).

[13] S. Faetti, Phys. Rev. A 36, 408 (1987).

[14] J.-B. Fournier and P. Galatola, Phys. Rev. E 60, 2404 (1999).

[15] G. Barbero and G. Durand, J. Phys. II 1, 651 (1991).

[16] A. Rapini and M. Papoular, J. Phys. (Paris) Colloq. 30, C4-54 (1969).

[17] P. Patricio, M. Tasinkevych, and M. M. Telo da Gama, Eur. Phys. J. E (in press).

[18] V. H. Schmidt, Phys. Rev. Lett. 64, 535 (1990).

[19] We thank M. Tasinkevych for helping us in obtaining this numerical result.

[20] L. D. Landau, E. M. Lifshitz, and L. P. Pitaevskii, Electrodynamics of Continuous media (ButterworthHeinemann, Oxford, 1984). 